\title{
Simplified method for estimating parasympathetic nerves activity by Lorenz plot of ECG RR intervals
}

\author{
Fumi Toyofuku ${ }^{1}$, Kazuhiko Yamaguchi ${ }^{1}$, Hiroshi Hagiwara ${ }^{2}$ \\ ${ }^{1}$ Graduate School of Science and Engineering, Ritsumeikan University, Shiga, Japan \\ ${ }^{2}$ College of information Science and Engineering, Ritsumeikan University, Shiga, Japan \\ rs029013@se.ritsumei.ac.jp
}

\begin{abstract}
Several studies have been made on that the power of the high frequency components (HF) from 0.15 to $0.40 \mathrm{~Hz}$ in heart rate reflects the parasympathetic nerves activity. However, there are some problems to apply HF to the evaluation index. For example, conversion from the time domain data into the frequency domain data needs complicated calculation. The purpose of this study is to show the evaluation method by Lorenz plot (LP) at electrocardiogram (ECG) RR intervals as a new evaluation index of the parasympathetic nerves activity. All the data of LP is projected on $y=x$ axis and $y=-x$ axis. In $y=x$ axis, standard deviation $\sigma(x)$ of the distance from the coordinate origin is calculated. In the same way, in $y=-x$ axis, standard deviation $\sigma(-x)$ is calculated. Ellipse area S showing variation of LP is $S=\pi \times \sigma(x) \times \sigma(-x)$. The correlationship of the significance probability $1 \%$ is shown between HF and LP's ellipse area S at ECG RR intervals. This indicates that LP's ellipse area S at ECG RR intervals is a reliable evaluation technique that takes the place of HF.
\end{abstract}

Keywords: Lorenz plot, electrocardiogram, RR intervals, high frequency components, parasympathetic nerves activity

\section{Introduction}

As for the modern society, it is said that the stressful society, and variety of mental stresses in the office and daily life have become problems. Relaxation that brings "Comfortable feeling" to relieve one's stress starts to garner attention in this modern society.

Several studies have been made on that the power of the high frequency components (HF) from 0.15 to 0.40 $\mathrm{Hz}$ in heart rate reflects the parasympathetic nerves activity $[1,2]$. However, there are some problems to apply HF to the evaluation index. For example, conversion from the time domain data into the frequency domain data needs complicated calculation. Because the Lorenz plot (LP) of electrocardiogram (ECG) RR intervals lets us perform the real-time evaluation, and also the analysis of the Lorenz plot is easier than that of HF. The purpose of this study is to show the evaluation method by Lorenz plot (LP) at ECG RR intervals as a new evaluation index of the parasympathetic nerves activity.

\section{Experimental method}

\subsection{Outline}

In experiment 1 and experiment 2 , five different modes of stimuli are given to the subjects for changing parasympathetic nerves activity [3].

Experiment 1: Several modes of massage, and posture (standing or sitting)

"Shiatsu (acupressure)", "Nipping", "Beating" of the massage, and "Standing with eyes closed "and "Sitting with eyes closed".
Experiment 2: Posture change stimulus

Degrees of backrest "90 degrees", "120 degrees", "150 degrees", and "180 degrees" and, "Eyes closed standing ".

\subsection{Subjects}

Experiment 1: ten subjects ( $21-23$ years old)

Experiment 2: eight subjects (21 - 24 years old)

The subjects take an adequate sleep and perform the experiment without immoderately eating and drinking after the previous night. In addition, the experimenter obtains informed consent from the subjects.

\subsection{Experimental protocol}

Each section enclosed with a square of the experiment 1 and 2 shown below is performed at random so that the order effect does not appear.

Experiment 1

- Standing with eyes closed $\downarrow$

- Sitting and eyes closed

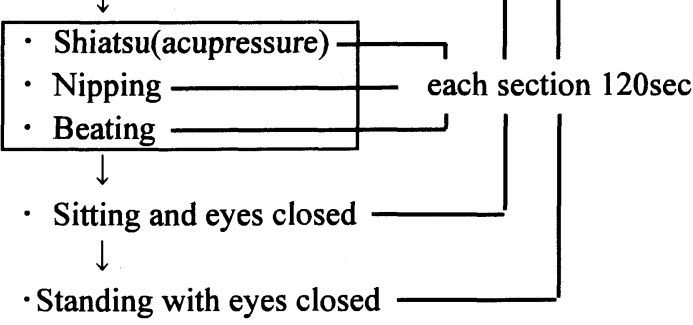




\section{Experiment 2}

- Standing with eyes closed $240 \mathrm{sec}$

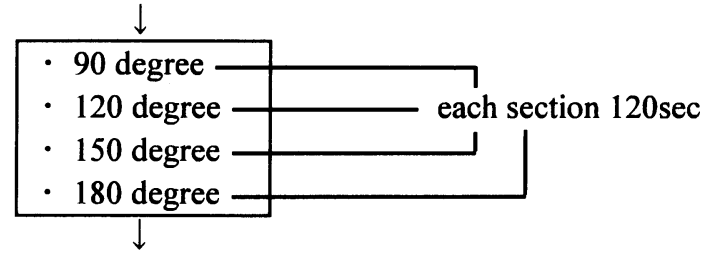

Standing with eyes closed $240 \mathrm{sec}$

\section{Analysis evaluation method}

\subsection{Method of calculating parasympathetic nerves activity (HF)}

The high frequency components (HF) is calculated by using the vital signal analysis program BIMUTAS II (KISSEI COMTEC Co. Ltd,). The electrocardiogram is measured by sampling frequency at $500 \mathrm{~Hz}$, and the noise is removed with high-pass filter $(0.1 \mathrm{~Hz})$ and also low-pass filter $(60 \mathrm{~Hz})$. Afterwards, the peak positions of ECG (RR intervals) are detected, and the value of HF is obtained by FFT (Fast Fourier Transform) of RR intervals' fluctuation (Fig.1, Fig.2).

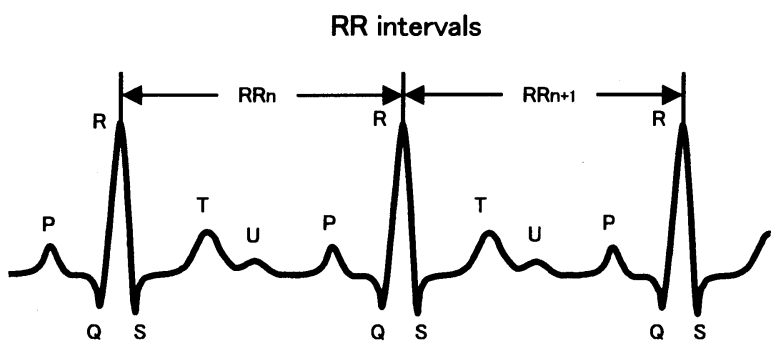

Fig.1 ECG RR intervals

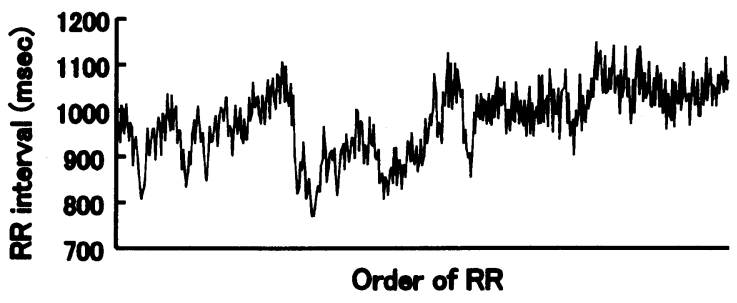

Fig.2 Fluctuation of RR intervals

It is said that the power of high-frequency component (HF: $0.15 \mathrm{~Hz}-0.40 \mathrm{~Hz}$ ) of the heart rate variability obtained by analyzing the ECG RR intervals will reflect the parasympathetic nerves activity. However, there are some problems to apply HF to the evaluation index (Fig.3). For example, conversion from the time domain data into the frequency domain data needs complicated calculation. Moreover, resampling of data is required when the number of data is too small, and real-time evaluation is difficult.

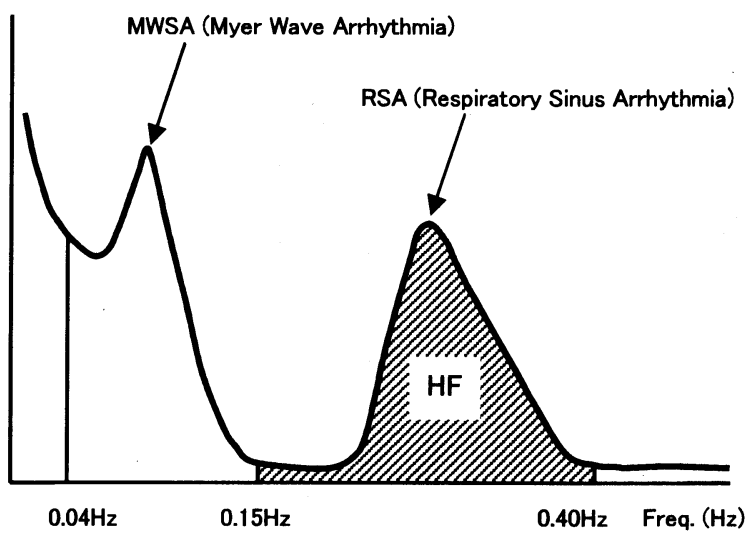

Fig.3 High-frequency component of the heart rate variability

\subsection{Evaluation by Lorenz plot at ECG RR intervals}

\subsubsection{Lorenz plot (LP)}

The Lorenz plot at ECG RR intervals is a figure where the $n^{\text {th }}$ ECG RR interval is plotted on the horizontal axis, and the $n+1^{\text {th }}$ ECG RR interval is plotted on the vertical axis [4, 5 and 6]. We propose the new evaluation index with LP at ECG RR intervals as substitute for HF.

\subsubsection{Advantage of LP}

There are some advantages of the use of LP to evaluate ECG RR intervals. For the first reason compared with the HF, the analysis method of LP is simple and is able to catch the change of heart rate in real time. For the second reason, the characteristics of the ECG RR intervals are visually and easily understood, and the noise elimination is easy. For these reasons, in LP at the electrocardiogram RR intervals, it is visually possible to analyze the change in the autonomic nervous activity in real time.

\subsubsection{Evaluation method by LP}

Figure 4 shows the evaluation method by LP at the ECG RR intervals. All the data of LP is projected to $y=$ $x$ axis and $y=-x$ axis.

In $y=x$ axis, standard deviation $\sigma(x)$ of the distance from the coordinate origin is calculated. In the same way, in $y=-x$ axis, standard deviation $\sigma(-x)$ of the distance from the coordinate origin is calculated. Ellipse area S showing variation of LP is $S=\pi \times \sigma(x) \times$ $\sigma(-\mathbf{x})$.

Ellipse areas S and HF calculated by the FFT (Fast Fourier Transform) are compared. 


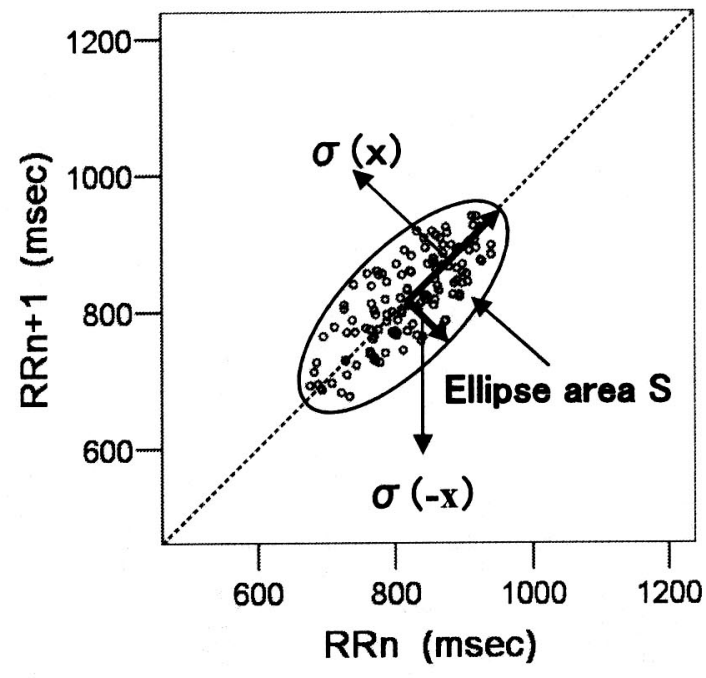

Fig.4 Evaluation method of LP

\section{Results}

\subsection{Changes of LP at ECG RR intervals by various stimulus}

Figure 5 shows LP in the standing position with eyes closed in experiment 1. Figure 6 shows LP in a sitting posture with eyes closed in experiment 1 .

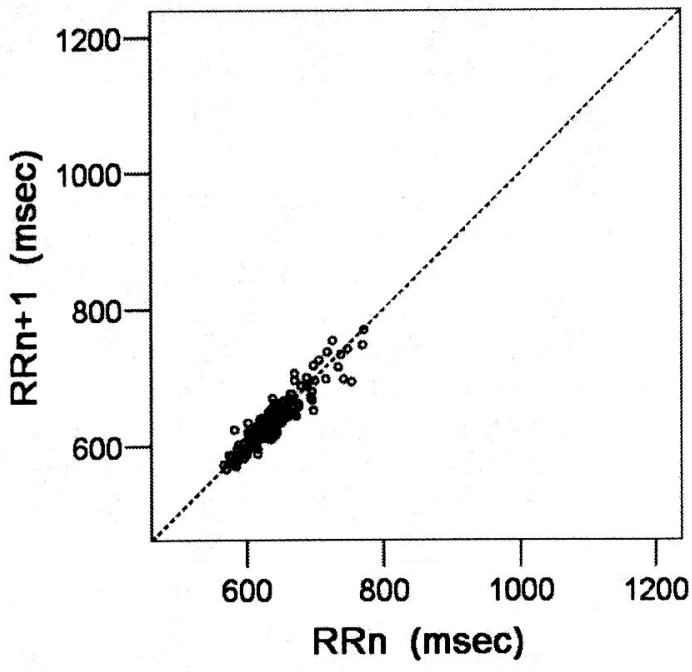

Fig. 5 Eyes closed at the standing position

In Figure 5, the ECG RR intervals chiefly change between $600 \mathrm{msec}$ and $800 \mathrm{msec}$, and are distributed around the $y=x$ axis. On the other hand, in Figure 6, the ECG RR intervals change between $800 \mathrm{msec}$ and $1000 \mathrm{msec}$, and are distributed a little wider compared with the standing position with eyes closed. In LP at ECG RR intervals, subjects' data can be observed visually and easily.

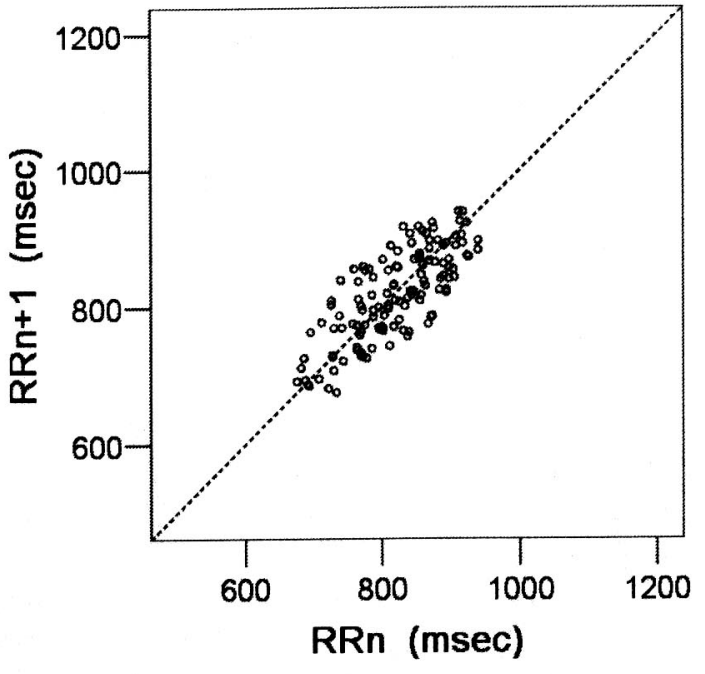

Fig.6 Eyes closed at a sitting posture

\subsection{Changes of HF and LP's ellipse area $S$}

Figure 7 and Figure 8 show the average and the standard deviation of HF and LP's ellipse area S at ECG RR intervals in each stimulus of experiment 1 and experiments 2. HF and LP's ellipse area S at ECG RR intervals show similar changes in experiment 1 and experiment 2. The relations between these two indices are analyzed.

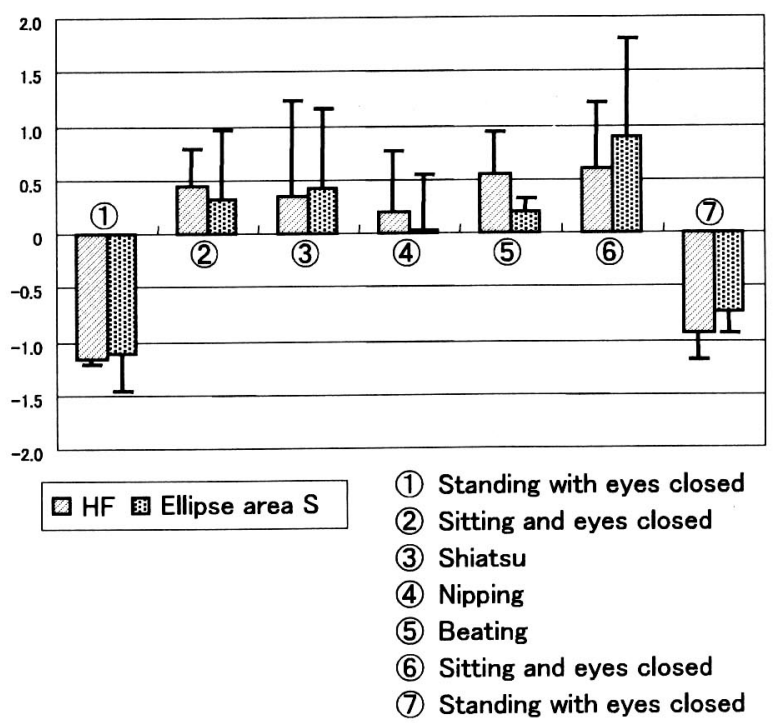

Fig.7 Changes of HF and area $S$ in experiment 1 


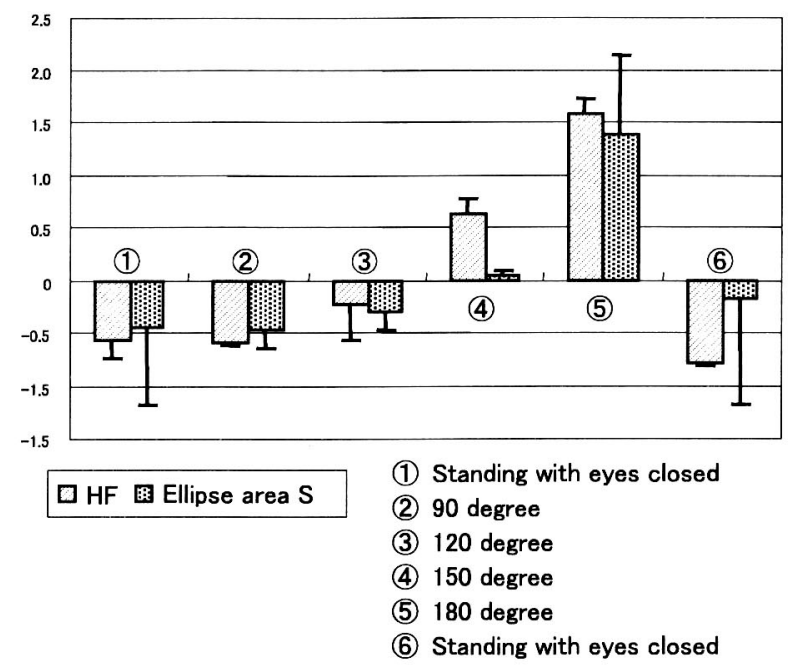

Fig. 8 Changes of $\mathrm{HF}$ and area $\mathrm{S}$ in experiment 2

\subsection{Correlationship of HF and LP's ellipse area $S$}

Table 1 shows the correlation coefficient of HF and LP's ellipse area $\mathrm{S}$ in experiment 1 and experiment 2 . There are statistically-significant relationships between HF and LP's ellipse area S.

Table1 Results of the correlationship

\begin{tabular}{|c|c|c|}
\hline & $\mathrm{n}=10$ & $\mathrm{n}=8$ \\
\hline & $\begin{array}{c}\text { Experiment 1 } \\
\mathrm{S}\end{array}$ & $\begin{array}{c}\text { Experiment 2 } \\
\mathrm{S}\end{array}$ \\
\hline $\mathrm{HF}$ & $0.606^{* *}$ & $0.527^{* *}$ \\
\hline
\end{tabular}

${ }^{* *} \mathrm{p}<0.01$

\section{Conclusion}

The correlationship of the significance probability $1 \%$ is shown between HF and LP's ellipse area $S$ at ECG RR intervals. This indicates that LP's ellipse area $S$ at ECG RR intervals is a reliable evaluation technique that takes the place of HF. Therefore, LP can evaluate the parasympathetic nerves activity easier than earlier methods.

In the future, it will be possible to monitor the stress factor and the autonomic nervous activity, etc. in real time.

\section{References}

[1] Yokoyama K., Kamiya N.: Simplified method for estimating high-frequency component of heart rate variability based on local characteristics in time series, The Japanese Journal of Ergonomics, 40(6):302-308, 2004
[2] Hayano J., Yamada M., Fujinami T., Yokoyama K, Watanabe Y., Takata K.: Spectral component of heart rate variability as an index of autonomic nervous function, Seibutsu Butsuri, 28(4): 198-202, 1988

[3] Yanmaguchi K., Toyofuku F., Mita Y., Takahashi Y., Hagiwara $H$. : A relationship between changes of contact pressure and subjective feelings, Proceedings of the Japan ergonomics society kansai branch : 61-64, 2005

[4] Yamazaki H., Hirakawa K.: Application of the Lorenz plot to analysis of time-series data, Technology reports of Kyushu University, 54(2):113-120, 1981

[5] Yamazaki H., Hirakawa K.: Application of the Lorenz plot to analysis of electrocardiographic rhythm - Measurement and analysis of the RR interval -, Technology reports of Kyushu University, 54(3):203-210, 1981

[6] Esperer HD., Esperer HC., Chernyak J.: Risk stratification in advanced heart failure using Lorenz plot indices of heart rate variability, Computers in Cardiology, 31: 209-212, 2004 